\title{
Effective diffusion constant in a two dimensional medium of charged point scatterers
}

\author{
D.S. Dean $\dagger \ddagger$ I.T. Drummond $\dagger$ and R.R. Horgan $\dagger$ \\ $\dagger$ DAMTP, CMS, University of Cambridge, Cambridge, CB3 0WA, UK \\ $\ddagger$ IRSAMC, Laboratoire de Physique Quantique, Université Paul Sabatier, 118 route \\ de Narbonne, 31062 Toulouse Cedex 04, France
}

\begin{abstract}
We obtain exact results for the effective diffusion constant of a two dimensional Langevin tracer particle in the force field generated by charged point scatterers with quenched positions. We show that if the point scatterers have a screened Coulomb (Yukawa) potential and are uniformly and independently distributed then the effective diffusion constant obeys the Volgel-Fulcher-Tammann law where it vanishes. Exact results are also obtained for pure Coulomb scatterers frozen in an equilibrium configuration of the same temperature as that of the tracer.

PACS numbers: 05.20.-y, 66.10.Cb, 66.30.Xj
\end{abstract}

\section{Introduction}

The bulk transport properties of random media are of great importance in physics and engineering. The computation of the bulk diffusivity, conductivity, permeability and dielectric constant from the statistical properties of their local fluctuations is referred to as homogenisation. These bulk quantities describe the transport properties over large length and time scales and will normally have well defined values when the statistics of the local fluctuations are homogeneous under translation. We will consider situations that are also statistically isotropic since a breakdown of isotropy involves further levels of complexity, important and interesting though they are.

In this paper we compute the effective diffusion constant for tracer particles travelling through a medium of fixed but randomly distributed centres of force. We we assume for simplicity that the centres of force are all identical but may have either positive or negative charge with equal probability. The tracer therefore experiences a randomly fluctuating potential with zero mean. This model of a disordered medium is a reasonable, if simplified, description of many real physical systems where the random potential is induced by impurities inserted in a regular background.

Many studies of diffusion in a random potential have been based on the assumption that it can be represented by a Gaussian field 1, 2, 3, 4, 5. Diffusion in a nonGaussian field has been studied using perturbation theory [6]. However the calculation is significantly more complicated than that for the Gaussian case. The significance of 
our investigation is that we obtain exact results for a class of problems, diffusion in random potentials of point scatterers, that are non-Gaussian and for which few results are known. The results are exact in one and two-dimensions but experience suggests that they may be indicative for higher dimensional cases.

\section{The Model}

The position $X_{i}$, of a particle, of negligible inertia, subject to a white noise, $w_{i}(t)$, and a potential $\phi(x)$, satisfies the equation

$$
\dot{X}_{i}=w_{i}(t)+\lambda_{0} \nabla_{i} \phi(X)
$$

where

$$
\left\langle w_{i}(t) w_{j}\left(t^{\prime}\right)\right\rangle=2 \kappa_{0} \delta_{i j} \delta\left(t-t^{\prime}\right)
$$

and $\langle\cdots\rangle$ denotes an average over the white noise. The Einstein relation implies that the local, or bare, diffusivity $\kappa_{0}$ and the coupling to the potential gradient $\lambda_{0}$ are related by the equation

$$
\frac{\lambda_{0}}{\kappa_{0}}=\beta=\frac{1}{T}
$$

where $T$ is the absolute temperature in appropriate units. The probability density, $p(x, t)$, for the position of the particle obeys

$$
\frac{\partial p(x, t)}{\partial t}=\kappa_{0} \nabla^{2} p+\lambda_{0} \nabla \cdot(p \nabla \phi) .
$$

The effective diffusivity, $\kappa_{e}^{(g)}$, appropriate to the dispersion of the particle at large times and distances is

$$
\kappa_{e}^{(g)}=\frac{1}{2 D} \lim _{t \rightarrow \infty} \frac{\left\langle X_{t}^{2}\right\rangle}{t}
$$

where $D$ is the dimension of space. The mean squared displacement of the particle is given by

$$
\left\langle X_{t}^{2}\right\rangle=\int d^{D} x x^{2} p(x, t)
$$

The superscript $g$ is used to denote the fact that we are considering the effective diffusion constant for a particle diffusing in a gradient field.

The description of the model is completed by specifying the structure of the potential $\phi(x)$ in terms of the scattering centres and their charges. In this paper we will consider potentials of the type

$$
\phi(x)=\sum_{n=1}^{N} q_{n} V\left(x-x_{n}\right),
$$

generated by $N$ scatterers frozen in the volume $V$. Here $q_{n}= \pm 1$ is the charge on the scatterer $n, x_{n}$ is its position and $V(x)$ the potential at the point $x$ due to a positively charged background particle at the origin. In this paper we shall consider distributions of the $x_{n}$ and $q_{n}$ such that the field $\phi$ is statistically invariant under global transformation 
$\phi \rightarrow-\phi$. This is clearly the case for distributions of the $x_{n}$ which are homogeneous and isotropic along with the condition that the distribution of the $q_{n}$ is invariant under the global charge transformation $q_{n} \rightarrow-q_{n}$. An example of this type of distribution is one where the positions $x_{n}$ are distributed uniformly and independently in the volume $V$ and the charges are taken to be independent and \pm 1 with probability $\frac{1}{2}$. In this case the disorder average is given by

$$
\langle\mathcal{O}\rangle_{d}=\prod_{n} \sum_{q_{n}= \pm 1} \frac{1}{2 V} \int d^{D} x_{n} \mathcal{O}
$$

One could also consider an strictly electroneutral ensemble where $N / 2$ of the scatterers have the charge \pm 1 , here the disorder average is thus

$$
\langle\mathcal{O}\rangle_{d}=\prod_{n} \frac{1}{V} \int d^{D} x_{n} \mathcal{O}
$$

we shall see however in the thermodynamic limit these two problems have the same diffusion constant. Another realisation of an ensemble of the type mentioned above is one where a system of $N / 2$ positive and negative charges, interacting via the Hamiltonian

$$
H=\sum_{i<j}^{N} q_{i} q_{j} V\left(x_{i}-x_{j}\right),
$$

are allowed to interact and equilibriate at some inverse temperature $\beta^{\prime}$, they are then frozen in this equilibrium configuration giving a distribution of the $x_{n}$ of

$$
P\left(\left\{x_{i}\right\}\right)=\frac{1}{Z\left(\beta^{\prime}\right)} \exp \left(-\beta^{\prime} \sum_{i<j}^{N} q_{i} q_{j} V\left(x_{i}-x_{j}\right)\right),
$$

where $Z\left(\beta^{\prime}\right)$ is the partition function for the system. Clearly the uniform distribution of equation (9) is recovered in the limit $\beta^{\prime} \rightarrow 0$.

\section{Associated permeability model}

In a previous paper we showed that there is a strong connection between our gradient flow model and the problem of computing the effective long range diffusivity, $\kappa_{e}^{(p)}$, of particle with a locally random diffusivity field $\kappa(x)$. A Langevin particle diffusing in a random medium with local diffusivity $\kappa(x)$ has a probability density which satisfies

$$
\frac{\partial p(x, t)}{\partial t}=\nabla \cdot \kappa(x) \nabla p
$$

In this case also the long time transport can also be partially described by the asymptotic behaviour of the mean squared displacement.

$$
\left\langle X_{t}^{2}\right\rangle \simeq 2 D \kappa_{e}^{(p)} t
$$

where $\kappa_{e}^{(p)}$ is the bulk or effective diffusivity of the medium.

The problem of calculating $\kappa_{e}^{(p)}$ is also equivalent calculating the bulk permeability of a porous medium, where the flow is described by Darcy's law [7, given that the local permeability is $\kappa(x)$. It is also equivalent to calculating the effective conductivity or 
effective dielectric constant in media where $\kappa(x)$ represents the local conductivity or dielectric constant. The superscript $p$ here is thus used to denote that the effective diffusion constant corresponds to an effective permeability problem. The problem of calculating $\kappa_{e}^{(p)}$ has been considered by numerous authors via exact relations in one and two dimensions [7, 8, 9, 10, and via perturbation techniques in three dimensions [1, 12, 13, 14.

The relevant connection between the permeability problem and the gradient flow problem emerges when the local diffusivity or permeability is chosen to be

$$
\kappa(x)=\kappa_{0} \exp (\beta \phi(x)) .
$$

In [15] it was shown that the bulk permeability or effective diffusion constant for equation (12) with a local diffusivity given by equation (14) and that for the gradient flow problem equation (41) with the same field $\phi$ are related by

$$
\frac{\kappa_{e}^{(g)}}{\kappa_{e}^{(p)}}=\frac{\kappa_{0}}{\bar{\kappa}},
$$

where

$$
\bar{\kappa}=\frac{1}{V} \int d^{D} x \kappa(x)
$$

The relation equation (15) holds in all dimensions where the corresponding $\kappa_{e}$ exist. In a statistically homogeneous system with short range correlations in the field $\phi$, in the limit of large $V$ we expect $\bar{\kappa}$ to be self averaging, or realisation independent, i.e.

$$
\bar{\kappa}=\langle\bar{\kappa}\rangle_{d}=\left\langle\frac{1}{V} \int d^{D} x \kappa(x)\right\rangle_{d}
$$

\section{Duality relation and the two dimensional result}

For two-dimensional systems we can obtain exact results by combining equation (15) with a standard duality theorem [8, 9, 10, For completeness we derive the relevant result in a form appropriate to the problem under consideration.

As a preliminary we note that when the fields $\phi(x)$ and $-\phi(x)$ are statistically equivalent it follows that local diffusivities $\kappa(x)$ and $\kappa^{\prime}(x)=\kappa_{0}^{2} / \kappa(x)$ are also statistically equivalent. We view equation (12) as describing the flow of the density $p(x)$ through the random medium. The associated current is $j(x)=\kappa(x) \nabla p(x)$. In the steady state we have

$$
\nabla \cdot(\kappa(x) \nabla p(x))=0
$$

and on averaging over the ensemble of samples we have, assuming a constant mean current and density gradient,

$$
\langle j(x)\rangle=\langle\kappa(x) \nabla p(x)\rangle=\kappa_{e}^{(p)}\langle\nabla p(x)\rangle .
$$

In two-dimensions equation (18) implies that there exists a dual field $\chi(x)$ such that

$$
j(x)=\kappa(x) \nabla p(x)=\kappa_{0} n \times \nabla \chi(x),
$$


where $n$ is a unit vector orthogonal to the two-dimensional plane of the problem. We also have the dual equation

$$
\kappa^{\prime}(x) \nabla \chi(x)=-\kappa_{0} n \times \nabla p(x)
$$

which implies

$$
\nabla \cdot\left(\kappa^{\prime}(x) \nabla \chi(x)\right)=0
$$

Since $\kappa^{\prime}(x)$ is statistically equivalent to $\kappa(x)$ it follows that

$$
\left\langle\kappa^{\prime}(x) \nabla \chi(x)\right\rangle=\kappa_{e}^{(p)}\langle\nabla \chi(x)\rangle,
$$

with the same effective permeability $\kappa_{e}^{(p)}$, as in the original problem. We have then the two dual results

$$
\kappa_{e}^{(p)}\langle\nabla p(x)\rangle=\kappa_{0} n \times\langle\nabla \chi(x)\rangle
$$

and

$$
\kappa_{e}^{(p)}\langle\nabla \chi(x)\rangle=-\kappa_{0} n \times\langle\nabla p(x)\rangle .
$$

The consistency of these equations implies that

$$
\kappa_{e}^{(p)}=\kappa_{0}
$$

Combining this result with equation (15) we obtain the general result valid in two dimensions

$$
\kappa_{e}^{(g)}=\frac{\kappa_{0}^{2}}{\bar{\kappa}}
$$

This equation (27) was previously used by the authors to solve the problem of calculating $\kappa_{e}^{(g)}$ in a homogeneous Gaussian field in two dimensions to give

$$
\kappa_{e}^{(g)}=\kappa_{0} \exp \left(-\frac{\beta^{2}}{2} \Delta(0)\right),
$$

where $\Delta$ is the connected two-point correlation function defined by

$$
\Delta\left(x-x^{\prime}\right)=\left\langle\phi(x) \phi\left(x^{\prime}\right)\right\rangle_{d}-\langle\phi(x)\rangle_{d}\left\langle\phi\left(x^{\prime}\right)\right\rangle_{d},
$$

the average again being over the sample disorder. This result is particularly interesting as it showed that the renormalisation group result for $\kappa_{e}^{(g)}$ [1, 2] in dimension $D$, given by

$$
\kappa_{e}^{(g)}(R G)=\kappa_{0} \exp \left(-\frac{\beta^{2}}{D} \Delta(0)\right),
$$

was exact in two dimensions. The equation (30) was already known to be exact in one dimension [2]. Indeed in one dimension the effective permeability is given for a general homogenous $\kappa(x)$ by

$$
\kappa_{e}^{(p)}=\left(\overline{\frac{1}{\kappa}}\right)^{-1}
$$

i.e. the harmonic mean of the local permeability. Using the result equation (15) we thus obtain the solution of the corresponding gradient flow problem in one dimension to be

$$
\frac{\kappa_{e}^{(g)}}{\kappa_{0}}=1 /\left(\bar{\kappa} \frac{\overline{1}}{\kappa}\right)
$$




\section{Some Examples}

For the problem of tracer particles moving through scatterers uniformly and independently distributed in two dimensions, we can combine equation (8) with equation (17) to obtain

$\bar{\kappa}=\prod_{n}\left[\int \frac{d^{2} x_{n}}{V} \frac{1}{2} \sum_{q_{n}} \exp \left(\beta q_{i} V\left(-x_{n}\right)\right)\right]=\left[1+\frac{1}{V} \int d^{2} x(\cosh (\beta V(x))-1)\right]^{N}$.

We now take the thermodynamic limit, that is $N \rightarrow \infty$ at fixed $\rho=N / V$ and obtain the exact result

$$
\kappa_{e}^{(g)}=\kappa_{0} \exp \left(-\rho \int d^{2} x(\cosh (\beta V(x))-1)\right) .
$$

The distribution given by equation (9) for an exactly neutral system of scatterers of density $\rho$ also leads to the same result equation (34) in the thermodynamic limit.

It is interesting to review the relationship of this result to equation (28) for the model in which $\phi(x)$ is a Gaussian field [1, 2] with zero mean. In the case of uniformly distributed randomly charged scatterers the two-point correlation function of the field $\phi$ is given by

$$
\Delta\left(x-x^{\prime}\right)=\rho \int d^{2} x^{\prime \prime} V\left(x-x^{\prime \prime}\right) V\left(x^{\prime}-x^{\prime \prime}\right) .
$$

Applying the Gaussian "prediction" equation (28) for the diffusivity therefore gives

$$
\kappa_{e}^{(g)}=\kappa_{0} \exp \left\{-\frac{\beta^{2}}{2} \rho \int d^{2} x V^{2}(x)\right\} .
$$

Comparing equations (36) and (34) we see that the result for the Gaussian field is recovered from that of the point scatterers in the limit $\beta \rightarrow 0$ while $\rho \beta^{2}=c$ with $c$ a constant. This limit where the scatterer disorder acquires a Gaussian character can also be found by examining the perturbation theory for this problem and holds in all dimensions. It is interesting to note that the Gaussian limit does not necessarily hold, as one may have naively expected from the central limit theorem, simply in the limit of large $\rho$.

It is illuminating to consider the particular case where the $V(x)$ is a screened Coulomb potential (Yukawa interaction) in two dimensions. We have

$$
\nabla^{2} V(x)-\mu^{2} V(x)=-2 \pi \delta(x)
$$

where $\mu^{-1}$ is the screening length. Writing $|x|=r, V(x)=V(r)$ has the asymptotic behaviour

$$
V(r) \propto \begin{cases}-\ln (r) & r \ll \mu^{-1} \\ \exp (-\mu r) / \sqrt{\mu r} & r \gg \mu^{-1}\end{cases}
$$

From this we see that the integral

$$
\int d^{2} x(\cosh (\beta V(x))-1)
$$

is convergent for large $r$ when $\mu>0$ and for small $r$ when $\beta<2$. The importance of the screening is now revealed since its removal, by setting $\mu=0$, leads to the integral 
becoming infra-red divergent thus forcing the diffusion constant to vanish. It would be interesting to investigate this regime separately since the actual behaviour of the sample would presumably be sub-diffusive and hence scale dependent.

The integral also diverges and the diffusivity goes to zero as $\beta$ approaches 2 from below, that is as the temperature cools to $T=1 / 2$. It is possible to analyse qualitative nature of the divergence since it is contained in the integral

$$
\int_{r \leq r_{0}} d^{2} x(\cosh (\beta V(x))-1) \simeq \int_{0}^{r_{0}} d r A r^{1-\beta} \simeq \frac{A}{2-\beta}
$$

for some $A>0$ and sufficiently small $r_{0}$. It follows that the diffusivity behaves as

$$
\frac{\kappa_{e}^{(g)}}{\kappa_{0}} \approx B \exp \left(-\frac{\rho A}{2-\beta}\right)
$$

which is of the form of the Volgel-Fulcher-Tammann law reported in fragile glass formers as they approach $T_{g}$. It is important to recognise that the effect is one associated with short distances. A qualitative light is shed on the circumstances by considering the equilibrium tracer particle density in the presence of the scattering centres. At any time only the nearest such scatterer dominates the tracer particle probability density. If this has the opposite sign of charge to the tracer particle then the probability density is $h(x)$ where

$$
h(x)=\frac{\exp (\beta V(x))}{\int_{V} d^{D} x^{\prime} \exp \left(\beta V\left(x^{\prime}\right)\right)},
$$

where we have placed the scatterer at the origin. Obviously this exhibits the same divergence as before and the density only exists for $\beta<2$. The physical interpretation of this result is that the tracer particle eventually becomes trapped at the point $x=0$ and thus diffusion is stopped.

We now consider a system where the configuration of the scatterers is that obtained by freezing an equilibrium configuration of mobile interacting scatterers at equilibrium at inverse temperature $\beta$, the same inverse temperature as the tracer particle 1.e. with a distribution of the $x_{n}$ given by equation (11) with $\beta=\beta^{\prime}$. This situation would apply to a very mobile tracer diffusing in a background of very slowly moving charges of the same valence, for example an electron in a background of immobile ions. To facilitate the calculation of $\bar{\kappa}$ we pass to the grand canonical ensemble for the scatters to obtain

$$
\begin{aligned}
\bar{\kappa}= & \frac{1}{\Xi} \sum_{N_{+}, N_{-}} \int \frac{d^{D} x_{i}}{N_{+} ! N_{-} !} \frac{d^{D} x}{V} \\
& z^{N_{+}+N_{-}} \exp \left(-\beta \sum_{i<j} q_{i} q_{j} V\left(x_{i}-x_{j}\right)\right) \exp \left(\beta \sum_{i} q_{i} V\left(x_{i}-x\right)\right),
\end{aligned}
$$

where the integration over $x$ above is the spatial averaging of $\kappa(x)$ over $V, \Xi$ is the grand partition function and $z$ is the fugacity of the positive and negative charges. The above expression can clearly be written as

$$
\bar{\kappa}=\frac{1}{z V \Xi} \sum_{N_{+}, N_{-}}\left(N_{-}+1\right) z^{N_{+}+N_{-}+1} Z_{N_{+}, N_{-}+1}
$$


where $Z_{N_{+}, N_{-}}$is the canonical partition function for a system of $N_{+/-}$positive/negative charges. In the thermodynamic limit we thus obtain

$$
\bar{\kappa}=\frac{\rho}{2 z},
$$

which yields

$$
\frac{\kappa_{e}^{(g)}}{\kappa_{0}}=\frac{2 z}{\rho}
$$

For the case of a pure Coulomb interaction i.e. when $\mu=0$

$$
V(x)=-\ln \left(\frac{|x|}{L}\right)
$$

where $L$ is a length scale which from here on we set to be 1 . The statistical mechanics of the two dimensional Coulomb gas has been recently exactly solved [16] in the region where the model is thermodynamically stable (i.e. $\beta<2$ ). For $\beta>2$ the system is thermodynamically unstable and collapses unless a hard core interaction is included between the particles; this instability is present for the reasons mentioned previously. In the stable region it has been shown

$$
\frac{\rho^{1-\beta / 4}}{z}=2\left(\frac{\pi \beta}{8}\right)^{\beta / 4} \frac{\Gamma(1-\beta / 4)}{\Gamma(1+\beta / 4)}\left[{ }_{2} F_{1}\left(\frac{1}{2}, \frac{\beta}{4-\beta} ; 1+\frac{\beta}{2(4-\beta)} ; 1\right)\right]^{1-\beta / 4}
$$

The resulting behaviour for $\kappa_{e}^{(g)}$ when $\beta \ll 1$ is

$$
\frac{\kappa_{e}^{(g)}}{\kappa_{0}} \approx(\rho \beta)^{-\beta / 4} \exp \left[-\left(2 \gamma+\ln \left(\frac{\pi}{2}\right)\right) \frac{\beta}{4}-\frac{7}{6} \zeta(3)\left(\frac{\beta}{4}\right)^{3}-\zeta(3)\left(\frac{\beta}{4}\right)^{4}\right],
$$

where ${ }_{2} F_{1}$ the the hyper-geometric function. Near the collapse point one finds

$$
\frac{\kappa_{e}^{(g)}}{\kappa_{0}} \approx\left(\frac{2-\beta}{\rho \pi}\right)^{1 / 2}
$$

as $\beta \rightarrow 2^{-}$. The interesting thing about these results is that the diffusivity is nonzero in all the region of stability, in contrast to the case of uniformly distributed Coulomb scatterers where it is zero in the thermodynamic limit in this region. Physically the fact that the Coulomb scatterers are allowed to equilibrate generates a screening characterised by a Debye length and the long range fluctuations of the electrostatic field are thus suppressed allowing a normal diffusion. The way in which the diffusion constant vanishes in equation (50) as $\beta \rightarrow 2^{-}$is also clearly very different to the case of a uniform distribution of Yukawa scatterers.

\section{Conclusion}

We have examined the problem of calculating the effective diffusivity of a Langevin particle diffusing in the force field generated by charged scatterers in two dimensions. The fact that the system is statistically electro-neutral means that the potential $\phi$ has the same distribution as $-\phi$. Using a general exact result connecting the effective diffusions constant of the gradient flow and varying permeability problem and an exact 
result in two dimensions for the permeability problem allows us to solve exactly the problem of calculating $\kappa_{e}^{(g)}$ in the cases studied here.

The results obtained give insight into the circumstances under which the random field may be taken to be Gaussian for the purposes of calculating the effective diffusion constant. It is clear from this study that the random field due to frozen scatterers will lead to transport properties that are generally different to that of a Gaussian random field with the same two point correlation function. It was also shown that the nature of the correlations between the point scatterers can drastically modify the behaviour of $\kappa_{e}^{(g)}$. For instance the diffusion constant for a system electro-neutral uniformly distributed Coulomb scatterers is always zero at finite temperature, however if they are taken to in an equilibrium configuration of the same temperature as the tracer particles then

$\kappa_{e}^{(g)}$ is finite at high temperature. The exact results here should be useful in developing perturbative techniques to treat problems in higher dimensions and in two dimensions where there is only one type of scattering particle, i.e. no charge.

\section{References}

[1] Dean D S, Drummond I T and Horgan R R 1994 J. Phys. A: Math Gen 275135

[2] Deem M W and Chandler D 1994 J. Stat. Phys. 76911

[3] Dean D S, Drummond I T and Horgan R R 1995 J. Phys. A: Math Gen 281235

[4] Dean D S, Drummond I T and Horgan R R 1995 J. Phys. A: Math Gen 286013

[5] Dean D S, Drummond I T and Horgan R R 1996 J. Phys. A: Math Gen 297867

[6] Drummond I T, Horgan R R and and da Silva Santos C A 1998 J. Phys. A: Math Gen 301341

[7] Matheron G 1967 Eléments pour une theorie des milieux poreux (Paris; Masson)

[8] Keller J B 1964 J. Math. Phys. 5548

[9] Dykhne A M 1971 Sov. Phys.-JETP 3263

[10] Dykhne A M 1971 Sov. Phys.-JETP 32348

[11] King P R 1987 J. Math. Phys. 203935

[12] Drummond I T and Horgan R R 1987 J. Phys. A: Math Gen 204661

[13] De Wit A 1995 Phys. Fluids 72553

[14] Abramovich B and Indelman P 1995 J. Phys. A: Math Gen 28693

[15] Dean D S, Drummond I T and Horgan R R 1997 J. Phys. A: Math Gen 30385

[16] Samaj L and Tavenec I 2000 J. Stat. Phys 101713 